\title{
Lossless Data Compression Based on Adaptive Linear Predictor for Embedded System of Unmanned Vehicles
}

\author{
FANGJIE YU \\ College of Information Science and Engineering, Qingdao Collaborative Innovation Center of Marine \\ Science and Technology, Ocean University of China, and Laboratory for Regional Oceanography and \\ Numerical Modeling, Qingdao National Laboratory for Marine Science and Technology, Qingdao, China

\section{LINHUA LI} \\ College of Information Science and Engineering, Qingdao Collaborative Innovation Center of Marine \\ Science and Technology, Ocean University of China, Qingdao, China \\ YANG ZHAO \\ College of Liberal Arts, Journalism and Communication, Ocean University of China, Qingdao, China

\section{MENGMENG WANG AND GUILIN LIU} \\ College of Information Science and Engineering, Qingdao Collaborative Innovation Center of Marine \\ Science and Technology, Ocean University of China, Qingdao, China

\section{GE CHEN} \\ College of Information Science and Engineering, Qingdao Collaborative Innovation Center of Marine \\ Science and Technology, Ocean University of China, and Laboratory for Regional Oceanography and \\ Numerical Modeling, Qingdao National Laboratory for Marine Science and Technology, Qingdao, China
}

(Manuscript received 29 December 2016, in final form 28 August 2017)

\begin{abstract}
Unmanned vehicles represent a significant technical improvement for ocean and atmospheric monitoring. With the increasing number of sensors mounted on the unmanned mobile platforms, the data volume and its rapid growth introduce a new challenge relative to the limited transmission bandwidth. Data compression provides an effective approach. However, installing a lossless compression algorithm in an embedded system, which is in fact limited in computing resources, scale, and energy consumption, is a challenging task. To address this issue, a novel self-adaptive lossless compression algorithm (SALCA) that is focused on the dynamic characteristics of multidisciplinary ocean and atmospheric observation data is proposed that is the extended work of two-model transmission theory. The proposed method uses a second-order linear predictor that can be changed as the input data vary and can achieve better lossless compression performance for dynamic ocean data. More than 200 groups of conductivity-temperature-depth (CTD) profile data from underwater gliders are used as the standard input, and the results show that compared to two state-of-the-art compression methods, the proposed compression algorithm performs better in terms of compression ratio and comprehensive power consumption in an embedded system.
\end{abstract}

\section{Introduction}

For modern marine and atmospheric observation platforms, unmanned vehicles play increasingly important roles for autonomous operations, such as ocean

Corresponding author: Ge Chen, gechen@ouc.edu.cn exploration, hazard response, and marine atmospheric boundary layer observation (Johnson et al. 2009: Reineman et al. 2013: Todd et al. 2016: Dooly et al. 2016). Unmanned vehicles, such as unmanned surface vehicles (USVs), underwater gliders, Argo floats, unmanned aerial vehicles (UAVs), and so on, are integrated with multisensors and transmit data in real time 
or near-real time primarily using satellite communication systems, which incur high cost and have limited bandwidth (André et al. 2015). Initially, a single sensor or a very limited number of sensors were mounted on mobile platforms; thus, little data were produced. However, as the rapid growth of research requirement, both the variety and the quantity of the sensors on the mobile platforms have increased. Take the Argo float for example: when the international Argo science team designed the criteria for the Argo array in 1998, only CTD sensors, which produce approximately $1 \mathrm{kB}$ of data for each profile, were mounted on Argo floats (Roemmich et al. 2004). Bishop et al. (2002) added carbon sensors to the Argo to detect organic carbon in the ocean. Körtzinger et al. (2005) added oxygen sensors to the platforms to perform biogeochemistry research. Riser and Johnson (2008) mounted dissolved oxygen sensors on the platforms to examine the balance of oxygen production in the North and South Pacific subtropical gyres. Perry et al. (2008) used optical sensors on underwater gliders to collect fluorescence and backscatter data in northeastern Pacific waters off the Washington coast, and Frajka-Williams et al. (2009) investigated the 2005 spring phytoplankton bloom in the Labrador Sea using a Seaglider equipped with hydrographic, bio-optical, and oxygen sensors. The size of the data produced by these sensors was approximately $100 \mathrm{kB}$ for each profile. To date, an increasing number of different types of sensors have been mounted on Argo and underwater gliders, including acoustic, biological, and optical sensors that represent mature technologies that can be used to monitor multiple ocean variables at unprecedented temporal and spatial resolutions (Johnson et al. 2009; Legler et al. 2015). The same could be found for other unmanned vehicles, such as USVs and UAVs (Biddle et al. 2015: Hodges and Fratantoni 2014).

The growing scientific demand has led to more sensors mounted on unmanned vehicles (André et al. 2015). As a result, more data will be produced and will be need to be transmitted by satellite, which will directly lead to three essential problems, that is, power consumption, communication cost, and real-time transmission possibility relative to limited bandwidth. Power consumption is a critical issue for the working life of unmanned vehicles. Communication, including transmission and reception, is one of the most power-consuming processes. It has been shown that approximately 3000 instructions could be executed for the same energy cost as sending 1 bit $1000 \mathrm{~m}$ by radio (Ephremides 2002), and reception power costs are approximately the same as transmission costs. The energy consumed by communication modules for data transmission and reception is much more than the energy consumed during data processing (Anastasi et al. 2009). As for communication, in recent years the Iridium and Argos satellite networks have primarily been used for data transmission (Legler et al. 2015). Considering the expensive communication costs and the limited bandwidth of satellite transmission, mass quantities of data will greatly increase the cost of the task. Meanwhile, the cost will block attempts to reduce transmission time on the surface, which is critical for the safety of the unmanned vehicles to lower the risk of theft, trawling, and collisions with vessels in high traffic areas, and to reduce the time of beaching on shores.

Data compression has been widely used to reduce data volumes of numerical model predications for efficient storage and transmission (Wang et al. 2015), and it has proven to be an efficient way to relieve the wireless communication burden. Therefore, it could play an important role in reducing communication cost, power consumption, and transmission time for unmanned vehicles. However, most unmanned vehicles use embedded systems as itscontroller. Compared with general purpose computers, typical embedded systems are limited in computing resources, small size, and low power consumption; thus, they are restricted by the complexity of lossless compression algorithms. In addition, realtime performance is required in most unmanned vehicles, which complicates the realization of such a method as well. There are two main groups of lossless compression algorithms suitable for embedded wireless communication. One is based on a dictionary. A dictionary-based lossless compression algorithm was first proposed by Jacob Ziv and Abraham Lempel and named LZ77 (Ziv and Lempel 1977). It provided fast compression speed with a low compression ratio, and it could be used in an embedded environment. An upgraded version of LZ77, the LZSS algorithm (Storer and Szmanski 1982),improves the compression performance when some special patterns occur frequently compared to all possible patterns (Sayood 2012, 270-272). However, the occurring patterns of marine observation data may cover all possible patterns, which results in a growing dictionary during compression. Consequently, the compression ratio decreases as the number of patterns increase. The other lossless compression method is based on entropy coding, which is more suitable for a data pattern with equal probability. The lossless entropy compression algorithm (Marcelloni and Vecchio 2008, 2009) is based on static Huffman coding. It exploits a short fixed dictionary whose size depends on the precision of an analog-to-digital converter. To track the change of data, a recently proposed two-modal transmission (TMT) approach (Huang and Liang 2007; Liang and Peng 2010) is an improved version of predictive coding. However, TMT is suitable only for stationary 


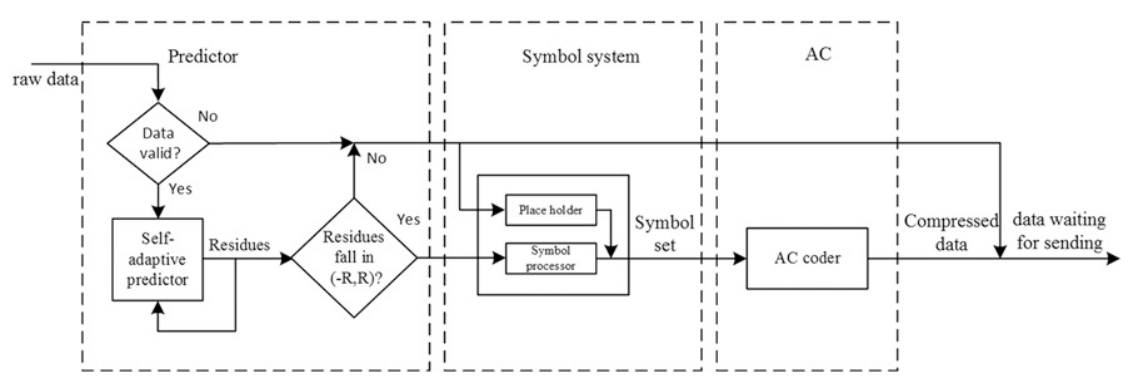

FIG. 1. System framework.

data. The ocean and atmosphere involve dynamic processes that change relative to many factors, such as time and depth. Therefore, ocean and atmospheric parameters change randomly in a small range, which is difficult to predict. These changes affect the prediction precision of TMT, and this, in turn, influences the compression ratio. For nonstationary data, which are common in marine observation, the performance of the predictor will not be efficient for long-term observation. Based on the TMT algorithm, we propose a self-adaptive lossless compression algorithm (SALCA) that is designed to improve prediction precision by adjusting the predictor according to the input to improve the compression ratio.

In this work, we focus on an efficient self-adaptive predictor that can adapt to a dynamic environment. Section 2 describes the compression method for data collected by marine devices, including the overall framework of the algorithm, the symbol system used in the data processing prior to compression by an arithmetic coding (AC) coder, and the adaptive linear predictive model to produce residues. Section 3 describes a series of experiments conducted to evaluate the compression ratio and the power consumption of the proposed algorithm. A summary of the compression algorithm and suggestions for future work are presented in section 4 .

\section{Theory and method}

As shown in Fig. 1, raw data from the sensor are processed by the self-adaptive module. A data checker is used to check the validity of the raw data. The difference value (DIF) of the data is defined as the difference between the current and previous data. If the DIF of the current data is greater than a threshold, which is an empirical value, then data are sent directly to the output data queue and a special defined symbol will be generated to indicate that the current data are invalid. Otherwise, the residues are divided into two parts based on the rule introduced in TMT. The symbol set is generated by the symbol processor and then sent to the AC coder.
The symbol system and AC coder in SALCA and TMT are the same, while the self-adaptive predictor in SALCA is different from the fixed one used in TMT. So, it is the performance of the predictors that affect the compression ratio of the two algorithms.

\section{a. Self-adaptive linear predictor}

\section{1) Prediction}

Considering the limited computational ability of the embedded system, the linear predictor was selected as the base model. For simplicity, the second-order linear predictor was used. For the series $x_{k}(k>2)$, the predicted item $\hat{x}_{k}$ is expressed as shown below:

$$
\hat{x}_{k}=c_{1} x_{k-1}+c_{2} x_{k-2}+c_{0},
$$

where both coefficients $c_{1}$, and $c_{2}$ and the constant term $c_{0}$ are determined by minimizing the square error of prediction $\hat{x}_{k}$ and $x_{k}$,

$$
e_{k}^{2}=\left(x_{k}-\hat{x}_{k}\right)^{2} .
$$

Here, $e_{k}^{2}$ can be considered as a function with variables $c_{1}, c_{2}$, and $c_{0}$ at time $k$.

\section{2) AdjustMent MECHANISM}

Figure 2 shows the curve of $e_{k}^{2}$ changing with $c$ (Sayood 2012, 270-272). According to the diagram, the optimum $c$ minimizes $d_{k}^{2}$. Here, we focus on the first partial derivative of $e_{k}^{2}$ over $c$. If $c$ is on the left side of the optimum value, $\partial e_{k}^{2} / \partial c$ will be negative. In addition, the greater the distance between $c$ and the optimum value, the greater the absolute value of $\partial e_{k}^{2} / \partial c$ will be. Thus, if $c$ is much less than the optimum value, the increment of $c$ should be large. Otherwise, the increment of $c$ should be small. If $c$ is on the right side of the optimum value, $\partial e_{k}^{2} / \partial c$ is positive. Coefficient $c$ should be decreased for the adjustment of $e_{k}^{2}$.

At any given moment, to adjust coefficients at time $k+1$, an increment can be added to the coefficient at $k$. The increment should be directly proportional to the 


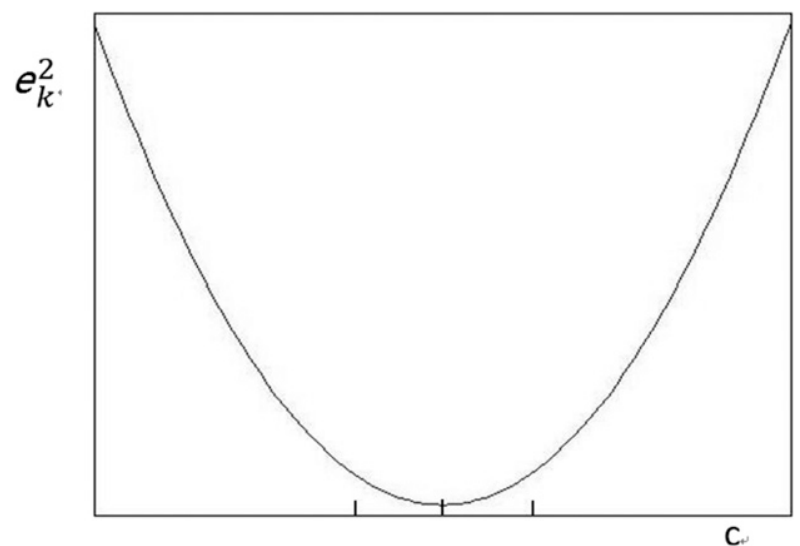

FIG. 2. Parameter adjustment.

differential coefficient of $e_{k}^{2}$ with the opposite sign, assuming that the adjusted coefficient is $c_{1}$ :

$$
c_{1}^{(k+1)}=c_{1}^{k}-\alpha \partial e_{k}^{2} / \partial c_{1},
$$

where step size $\alpha$ is a direct constant,

$$
\begin{aligned}
& \partial e_{k}^{2} / \partial c_{1}=-2\left(x_{k}-c_{1} x_{k-1}-c_{2} x_{k-2}-c_{0}\right) x_{k-1}, \\
& \partial e_{k}^{2} / \partial c_{1}=-2 e_{k} x_{k-1} .
\end{aligned}
$$

By substituting (2) into (1), we obtain

$$
c_{1}^{(k+1)}=c_{1}^{k}+2 \alpha e_{k} x_{k-1} .
$$

In the same manner, for $c_{2}$ and $c_{0}$ we obtain

$$
\begin{aligned}
& c_{2}^{(k+1)}=c_{2}^{k}+2 \alpha e_{k} x_{k-2}, \\
& c_{0}^{(k+1)}=c_{0}^{k}+2 \alpha e_{k} .
\end{aligned}
$$

Thus, coefficients $c_{0}, c_{1}$, and $c_{2}$ can be adjusted automatically by the change of input data. However, it is difficult to select a proper step size $\alpha$ to guarantee the stability of the coefficient adjustment. According to the literature (Gui and Adachi 2013), $\alpha$ can be replaced by

$$
\alpha=\left(\frac{1}{2}\right)\left\{\alpha_{1} /\left[\mathbf{x}^{\mathrm{T}}(t) \mathbf{x}(t)\right]\right\}
$$

Note that $\mathbf{x}(t)$ is the vector of $\left(1, \mathbf{x}_{k-1}, \mathbf{x}_{k}\right)^{\mathrm{T}}$ and $\alpha_{1}$ is constant and is usually set to 1 (Gui and Adachi 2013).

\section{b. Coding system}

In the proposed algorithm, the coding system is the common part of SALCA and TMT. The coding system consists of two parts, symbol system and AC coder.

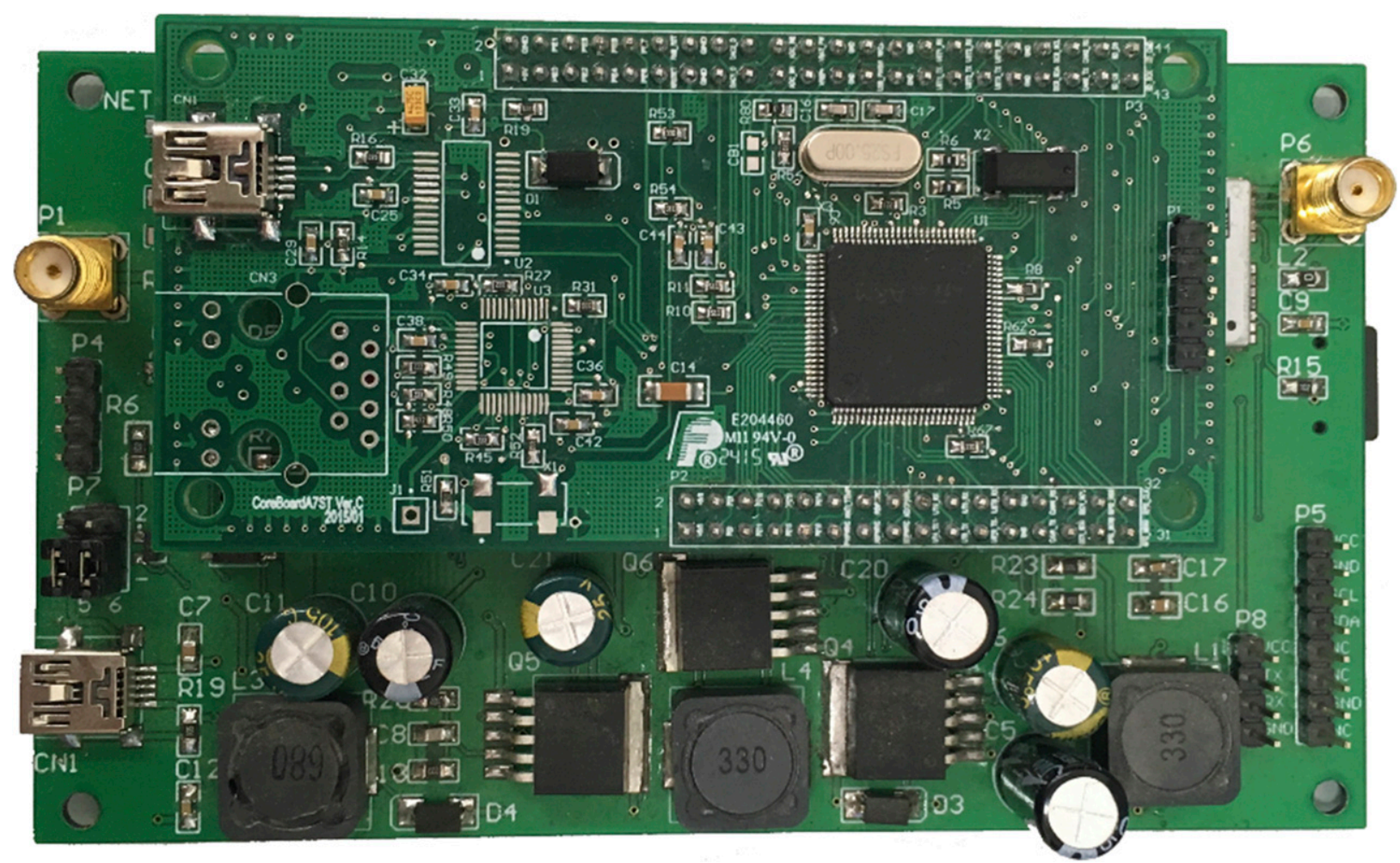

FIG. 3. Cortex-M4 hardware platform. 

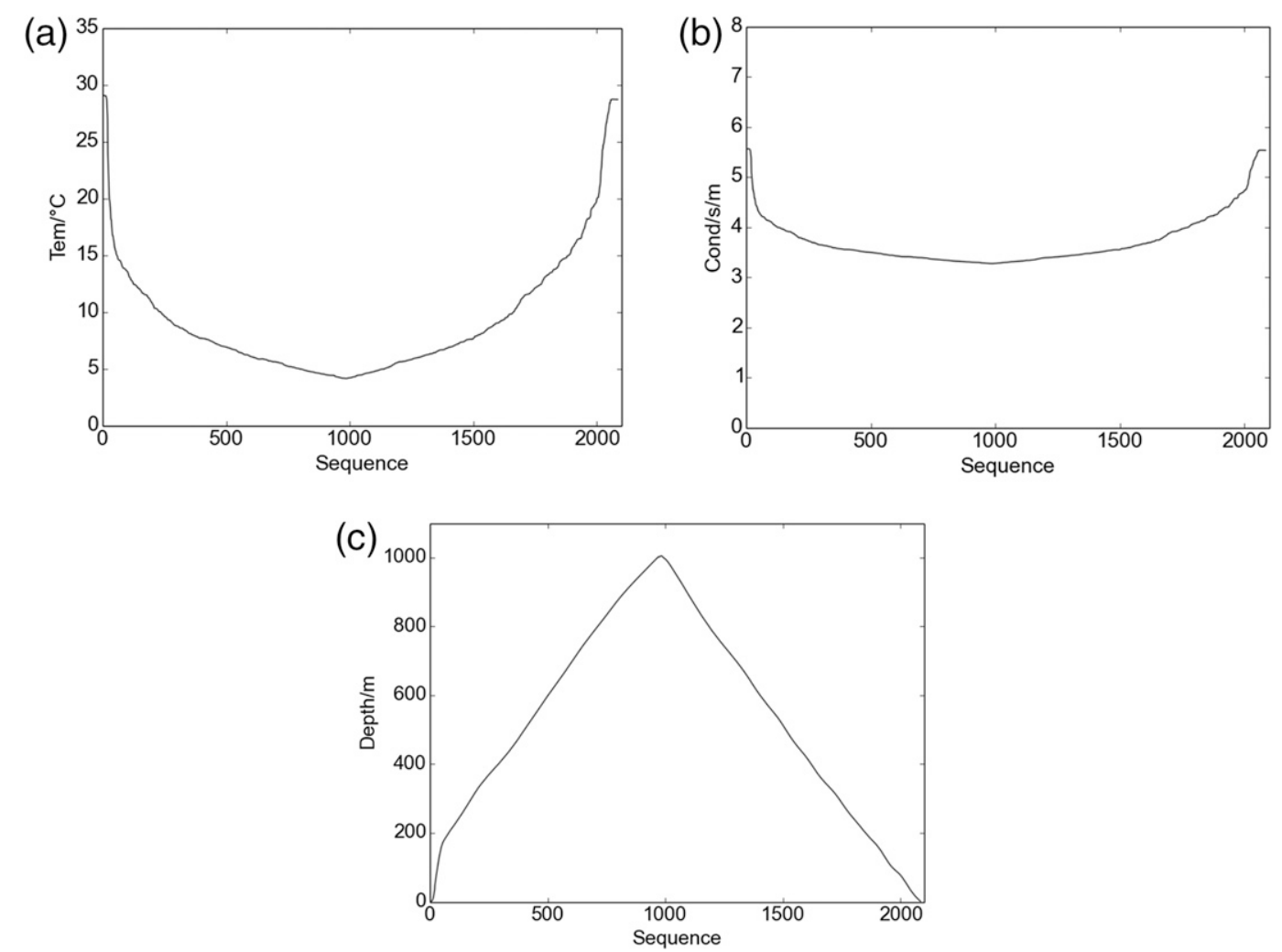

FIG. 4. CTD data obtained from glider (depth ranges from 1.0 to $1000.0 \mathrm{~m}$ ): (a) temperature, (b) conductivity, and (c) depth.

\section{1) SYMBOL SYSTEM}

The data from sensors are always a large integer sequence, and every integer in the sequence can be different. For example, an integer that consists of four numbers has 10000 possibilities. It is impossible for memory-limited platforms to compress such a large letter set. Thus, the data sequence should be processed before being sent to the AC coder. The raw data collected from sensors on Argo or a glider are integers rather than floating-point numbers. For example, CTD temperature data are 23568 rather than 23.568. When data are compressed, each integer should be separated into a character sequence according to the M-base; " 3345 " is separated into $\left\{3^{\prime}, 3^{\prime},{ }^{\prime} 4^{\prime}, 5^{\prime}\right\}$ according to decimal and $\left\{{ }^{\prime},{ }^{\prime}, \mathrm{D}^{\prime},{ }^{\prime} 1^{\prime},{ }^{\prime} 1^{\prime}\right\}$ according to hexadecimal. If the data value is negative-for example, "-3345," -then the sequence will be $\left\{{ }^{\prime}-, 3^{\prime}, 3^{\prime}, 4^{\prime}, 5^{\prime}\right\}$ according to decimal (Liang and Peng 2010).

Since the residues satisfy Laplacian distribution, approximately $98.56 \%$ of the residues fall inside the range $[-3 \sigma, 3 \sigma]$ with zero mean. The number of some large residues that fall outside the range $[-3 \sigma, 3 \sigma]$ will be ignored. Since all residues should be encoded, the symbol set can be very large as a result of some large residues. Therefore, if the terms of the residual sequence are within the range $[-R, R](R>0 ; R$ is the threshold value), then they will be coded; otherwise, they are not processed by coder. The receiving side will decode the sequence according to the same rule. Value $R$ and M-base are calculated at the receiving side and are sent to the platform prior to executing the compression process.

Some of the residues may not be separated into a character sequence if they are not in the range $[R,-R]$. Additional symbols are needed to hold the place for residues not in range. In addition, an invalid data placeholder is also required to hold the position. Note that a special symbol is encoded to mark the end of coding. Thus, there should be $\mathrm{M}+3$ symbols for the coding system. And, two types of data are sent back, that is, a decode sequence and a coded sequence. When the decoder decodes the additional symbol, it will search the decoded sequence to replace the symbol.

\section{2) AC CODER}

An AC coder is used in the proposed algorithm. The AC coder is based on the implementation described by Witten et al. (1987). To obtain better performance, the 

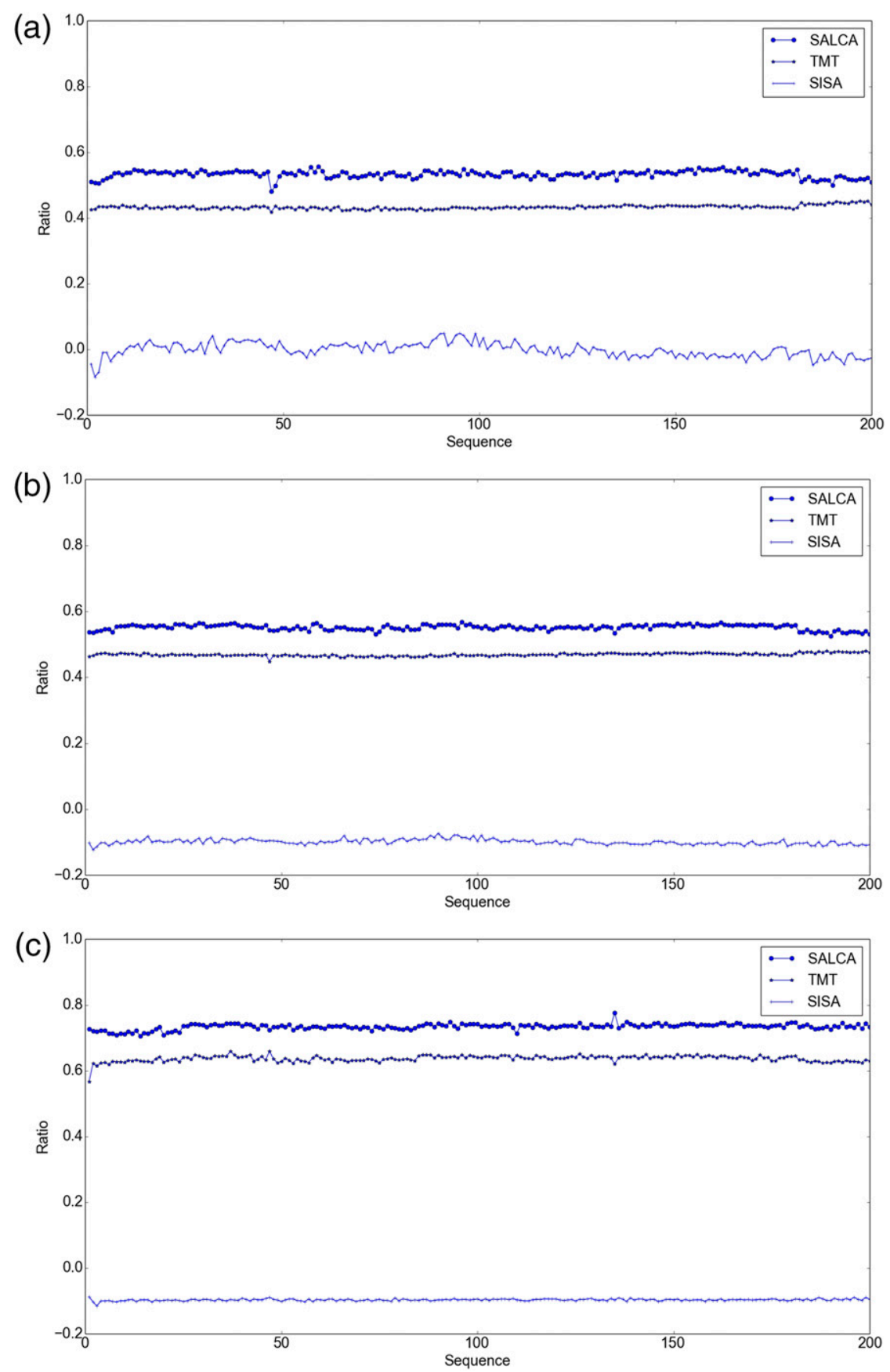

FIG. 5. Compression ratio of three types of data (200 groups): (a) temperature, (b) conductivity, and (c) depth.

dynamic symbol set introduced by Sayood (2012, 270-272) was used to replace the static one.

\section{Experiment and data analysis}

To get a comprehensive evaluation of the proposed algorithm, several experiments were designed to test performance. The STM32F4 series processors, which are based on an Advanced RISC Machine (ARM) Cortex-M4 32-bit reduced instruction set computing (RISC) core operating at $168 \mathrm{MHz}$, were used in the experiments as the embedded processor. Because of their high performance and low power consumption, the ARM series processors have been widely used in many unmanned vehicles as 
embedded control systems (Yu et al. 2011). The onboard RAM is $192 \mathrm{kB}$. Figure 3 shows the self-designed hardware platform running the algorithms. The Iridium 9602 module was used to transfer data via satellite.

The data used in the experiment were collected by the Sea-Wing underwater glider developed by the Shenyang Institute of Automation, Chinese Academy of Sciences (Yu et al. 2011). In May 2015, a one-month mission was conducted in the South China Sea from east to west along the continental slope. CTD cells were mounted on the glider. These cells sampled every $6 \mathrm{~s}$ during the diving profile and the climbing profile. The diving depth of the glider was $1000 \mathrm{~m}$. During the experiment, more than 200 profiles were collected. Figure 4 shows the CTD data changes for one dive (two profiles).

\section{a. Comparison of compression ratio}

More than 200 groups of field observation CTD data were compressed by SALCA, TMT, and LZSS, and details about the 200-group compression ratio of the three algorithms are shown in Fig. 5. The average compression ratios of each compression algorithm were calculated, and the results are shown in Table 1.

For TMT and SALCA algorithms, the compression ratio rankings of the three kinds of data (temperature, conductivity, depth) are nearly the same, which shows different data patterns could affect the compression ratio. The reason why the compression ratio of depth is highest could be that the data change of depth presents more regularly (Fig. 4) compared to temperature and conductivity. However, LZSS shows a small difference in compression ratios (within 10\%), which indicates that it is not as sensitive to data patterns with equal probability as the abovementioned two methods.

Meanwhile, SALCA shows a higher compression ratio than TMT in all cases, while LZSS demonstrates the lowest compression ratio. We evaluated why SALCA outperformed the other algorithms. As can be seen in Fig. 4, the observed data changed significantly in some layers that were known as the thermocline or the halocline. A sharp falling edge (rising edge) of temperature and conductivity could be seen in the diving profile (climbing profile). Because of the invariant feature of the parameters of the TMT algorithm, these rapid changes would cause the predictor to fail tracking the variation. As a result, some large residual values would be produced. However, for SALCA, the output parameters of the self-adaptive linear predictor could change with the rapid change of input data. Thus, as seen in Fig. 6, both temperature and conductivity residue distributions of SALCA are more concentrated than those of TMT. Note that as the residue distribution becomes more concentrated, fewer
TABLE 1. Compression ratio

\begin{tabular}{lccr}
\hline \hline \multicolumn{1}{c}{ Sensor } & SALCA & TMT & LZSS \\
\hline Temperature & $52.70 \%$ & $40.13 \%$ & $0.40 \%$ \\
Conductivity & $55.14 \%$ & $46.70 \%$ & $-9.82 \%$ \\
Depth & $73.16 \%$ & $63.79 \%$ & $-9.23 \%$ \\
\hline
\end{tabular}

symbols are required, which will directly improve the compression ratio.

For depth, the CTD sensor mounted on the glider platform was set to sample at a fixed time interval. The glider moves at nonuniform speed in different layers of the ocean; thus, the depth interval is not the same. These irregular variations challenge the fixed-parameter predictor of TMT, while SALCA shows more adaptability because of the self-adaptive linear predictor. Similar results for the residue distribution of depth between TMT and SALCA are shown in Fig. 6.

\section{b. Comparison of power consumption}

As mentioned previously, compression power consumption is a key factor in embedded systems, such as gliders, AUVs, etc. To evaluate the power consumption performance of the three algorithms, we calculated the total energy consumption incurred by both the data compression and data transmission processes.

First, power consumption tests for the three lossless compression algorithms (SALCA, TMT, and LZSS) were carried out. Approximately 200 groups of CTD data were used as input data. The amount of data transferred is the number of bytes after compression, and these data are sent directly to the satellite communication module. The compression power consumption of each compression algorithm is measured separately in the compression process. As shown in Table 2, the power consumption of SALCA and TMT are approximate and both are lower than LZSS. The temperature and depth data compression power consumption of SALCA is a little higher than TMT, but its conductivity data compression power consumption is approximate to TMT. Taking temperature data as an example, for the same amount of raw data (935298 bytes), the compressed result of the LZSS algorithm was 931599 bytes and the power consumption was 17.001 J, the TMT algorithm was 559987 bytes and $15.701 \mathrm{~J}$, and the SALCA algorithm was 442409 bytes and $16.512 \mathrm{~J}$. Thus, compared to the TMT algorithm, the SALCA algorithm improved the compression ratio (about $12.6 \%$ ) while increasing power consumption (about 5.2\%). Similar results can be seen in depth, which indicates the reason for better compression performance of SALCA is at the cost of power consumption. However, conductivity 
(a)

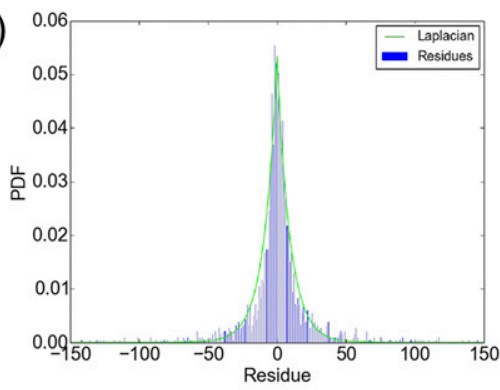

(c)

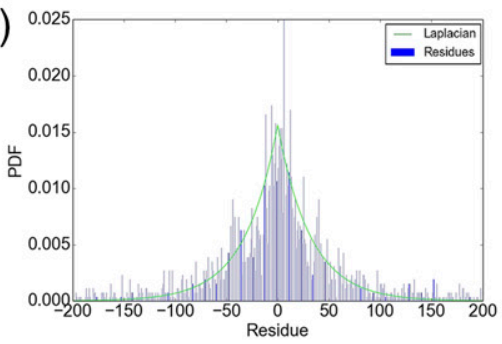

(e)

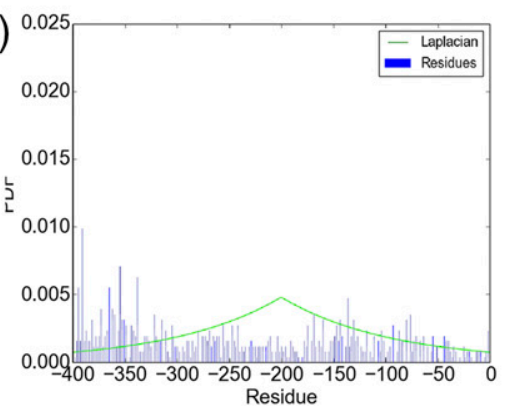

(g)

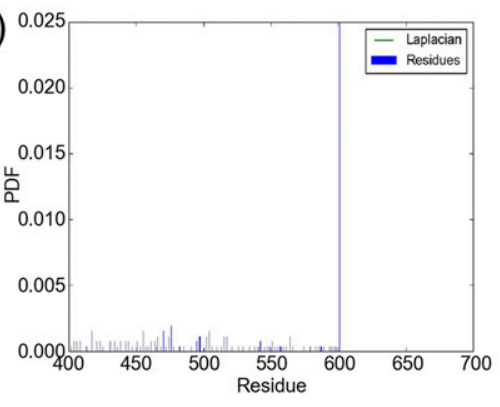

(b)

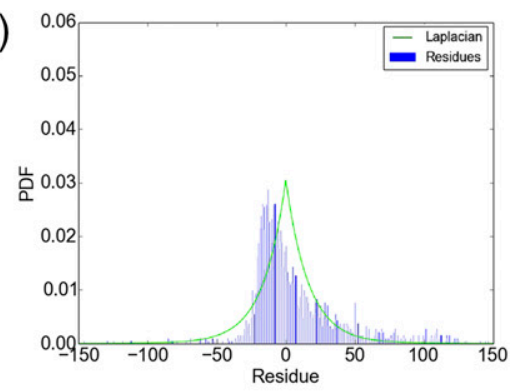

(d)

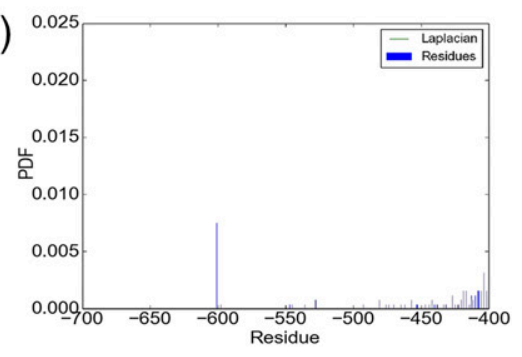

(f)

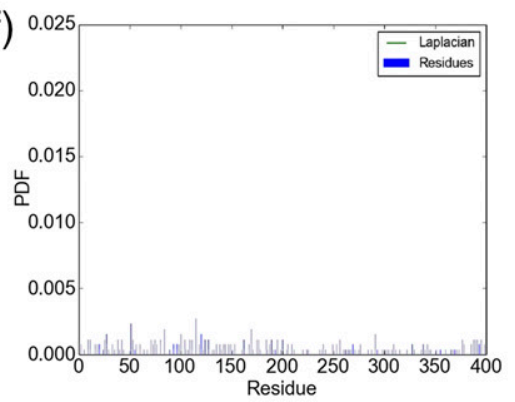

(h)

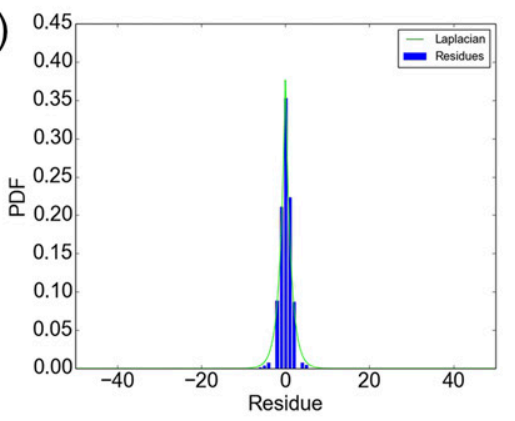

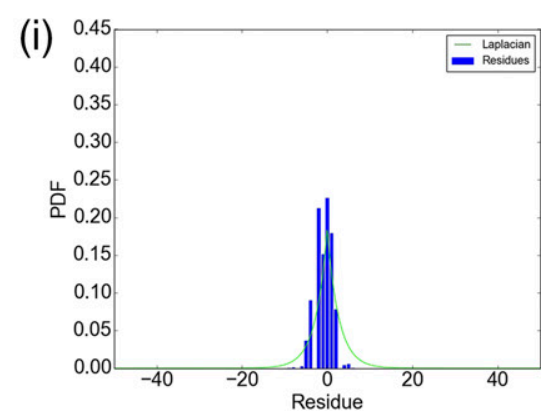

FIG. 6. (a) Temperature distribution of SALCA residues with Laplacian distribution $(\sigma=13.21)$. (b) Temperature distribution of TMT residues with Laplacian distribution $(\sigma=23.23)$. (c) Conductivity distribution of SALCA residues with Laplacian distribution $(\sigma=45.23)$. (d)-(g) Conductivity distribution of TMT residues with Laplacian distribution $(\sigma=148.33)$. (h) Distribution of SALCA residues with Laplacian distribution $(\sigma=1.85)$. (i) Distribution of TMT residues with Laplacian distribution $(\sigma=3.84)$. 
TABLE 2. Total power consumption for compression (200 groups of data).

\begin{tabular}{lccc}
\hline \hline Predictor & SALCA $(\mathrm{J})$ & TMT $(\mathrm{J})$ & LZSS $(\mathrm{J})$ \\
\hline Temperature & 16.512 & 15.701 & 17.001 \\
Conductivity & 21.620 & 21.664 & 22.728 \\
Depth & 14.268 & 13.187 & 18.560 \\
\hline
\end{tabular}

shows a different result, so it is meaningful to find out the reason. Table 3 shows the total prediction and coding power consumption of SALCA and TMT. During prediction the power consumption of SALCA is higher than TMT, while in coding the power consumption of SALCA is lower than TMT. Figure 6 and Table 3 show that the more concentrated residues present in the prediction process, the less power consumption there will be in the coding procedure. The phenomenon that SALCA's power consumption of conductivity is a little lower than TMT's is understandable. Using adaptive prediction, the distribution of the residue distribution can be more concentrated, thereby reducing implementation time and reducing the power consumption of data compression.

Second, the power consumption of satellite transmission was considered. The Iridium 9602 satellite transmission module was used to send compressed data, and the power consumption of this module is shown in Table 4. The compressed data of the three algorithms were transmitted using the Iridium module while measuring the power consumption of the transmission process. The results are shown in Table 5. As can be seen, the satellite transmission power is proportional to the size of the data sent; that is, as more bytes are sent, power consumption increases.

Third, the total power consumption was calculated by summing the data compression power consumption and satellite transmission power consumption. The consumption results for each algorithm are consistent, and data compression power consumption represents only $1 \%$ of total power consumption. Using the SALCA algorithm as an example, compared to satellite transmission power consumption, data compression power consumption was very small, that is, only $1 \%$ of the total power consumption. This indicates that satellite

TABLE 3. Total prediction and coding power consumption of SALCA and TMT (200 groups of data).

\begin{tabular}{|c|c|c|c|c|}
\hline & Predictor & & Coding & \\
\hline Predictor & SALCA (J) & TMT (J) & SALCA (J) & TMT (J) \\
\hline Temperature & 12.016 & 9.795 & 4.536 & 5.906 \\
\hline Conductivity & 14.153 & 11.852 & 7.466 & 9.812 \\
\hline Depth & 11.739 & 9.420 & 2.526 & 3.785 \\
\hline
\end{tabular}

TABLE 4. Iridium 9602 parameters.

\begin{tabular}{ccc}
\hline \hline $\mathrm{U}(\mathrm{V})$ & $\mathrm{I}(\mathrm{mA})$ & Speed $\left(\right.$ bytes s $\left.^{-1}\right)$ \\
\hline 5 & 190 & 307.2 \\
\hline
\end{tabular}

transmission incurs the highest power consumption. Thus, satellite data transmission length should be compressed to most effectively reduce total system power consumption. On the other hand, for temperature data, the lengths of the compressed data for SALCA, TMT, and LZSS were 442 409, 559987, and 931599 bytes, respectively. SALCA saved 117578 bytes compared to TMT, 489190 bytes compared to LZSS, and 492889 bytes compared to the uncompressed data. As a result, when compressed data were transmitted by Iridium, the proposed SALCA algorithm saved 362.784, 1513.274, and $1507.722 \mathrm{~J}$, respectively. In other words, compared to the TMT and LZSS algorithms, SALCA can save $21 \%$ and $52 \%$ power consumption, respectively.

\section{Conclusions}

We proposed a lossless adaptive data compression algorithm based on TMT for the mutative marine observation data. The experiments have shown that the technique achieves good compression performance for CTD data obtained by glider. The major difference between the proposed data compression algorithm and TMT is that SALCA uses the adaptive liner predictor to track the change of the data during compression. For marine data compression, using adaptive liner predictor, the proposed compression algorithm can achieve better data compression than TMT. It has been demonstrated that a $52 \%$ or higher compression ratio can be achieved for the CTD data obtained by glider. A better compression ratio can reduce the communication time that Argo floats and a glider spends on the surface, thus lowering the risk of being stolen or trawled. The numerical experiments conducted in this research have shown the power consumption performance of each algorithm. The results of the numerical experiment also indicate that the proposed algorithm is better at energy saving. In the future, we plan to develop a multitype data compression algorithm using a type-fusion adaptive predictor that can process multitype data at the same time.

TABLE 5. Power consumption of data transmission.

\begin{tabular}{lccc}
\hline \hline Predictor & SALCA $(\mathrm{J})$ & TMT $(\mathrm{J})$ & LZSS $(\mathrm{J})$ \\
\hline Temperature & 1368.127 & 1731.731 & 2880.921 \\
Conductivity & 1938.250 & 2302.674 & 4744.285 \\
Depth & 769.744 & 1038.382 & 2868.648 \\
\hline
\end{tabular}


Acknowledgments. This research was jointly supported by the following programs: 1) the National Natural Science Foundation of China under Grants U1606405, 41527901, and 61361136001; 2) the Qingdao National Laboratory for Marine Science and Technology under Grant QNLM2016ORP0105; 3) the Fundamental Research Funds for the Central Universities under Grant 201562024; 4) Qingdao science and technology projects of applying basic research for young under Grant 14-2-4-92-jch., and 5) The National High Technology Research and Development Program of China under Grant 2014AA09A511.

\section{REFERENCES}

Anastasi, G., M. Conti, M. Di Francesco, and A. Passarella, 2009: Energy conservation in wireless sensor networks: A survey. Ad Hoc Networks, 7, 537-568, https://doi.org/10.1016/ j.adhoc.2008.06.003.

André, X., B. Moreau, and S. Le Reste, 2015: Argos-3 satellite communication system: Implementation on the Arvor oceanographic profiling floats. J. Atmos. Oceanic Technol., 32, 1902-1914, https://doi.org/10.1175/JTECH-D-14-00219.1.

Biddle, L. C., J. Kaiser, K. J. Heywood, A. F. Thompson, and A. Jenkins, 2015: Ocean glider observations of iceberg-enhanced biological production in the northwestern Weddell Sea. Geophys. Res. Lett., 42, 459-465, https://doi.org/10.1002/2014GL062850.

Bishop, J. K. B., R. E. Davis, and J. T. Sherman, 2002: Robotic observations of dust storm enhancement of carbon biomass in the North Pacific. Science, 298, 817-821, https://doi.org/ 10.1126/science.1074961.

Dooly, G., and Coauthors, 2016: Unmanned vehicles for maritime spill response case study: Exercise Cathach. Mar. Pollut. Bull., 110, 528-538, https://doi.org/10.1016/j.marpolbul.2016.02.072.

Ephremides, A., 2002: Energy concerns in wireless networks. IEEE Wireless Commun., 9, 48-59, https://doi.org/10.1109/ MWC.2002.1028877.

Frajka-Williams, E., P. B. Rhines, and C. C. Eriksen, 2009: Physical controls and mesoscale variability in the Labrador Sea spring phytoplankton bloom observed by Seaglider. Deep-Sea Res. I, 56, 2144-2161, https://doi.org/10.1016/j.dsr.2009.07.008.

Gui, G., and F. Adachi, 2013: Improved least mean square algorithm with application to adaptive sparse channel estimation. EURASIP J. Wireless Commun. Networking, 2013, 204, https://doi.org/10.1186/1687-1499-2013-204.

Hodges, B. A., and D. M. Fratantoni, 2014: AUV observations of the diurnal surface layer in the North Atlantic salinity maximum. J. Phys. Oceanogr., 44, 1595-1604, https://doi.org/ 10.1175/JPO-D-13-0140.1.

Huang, F., and Y. Liang, 2007: Towards energy optimization in environmental wireless sensor networks for lossless and reliable data gathering. Proc. IEEE Int. Conf. on Mobile Adhoc and Sensor Systems (MASS 2007), Pisa, Italy, IEEE, 6 pp., https://doi.org/10.1109/mobhoc.2007.4428745.

Johnson, K. S., W. M. Berelson, E. Boss, Z. Chase, H. Claustre, S. R. Emerson, and N. Gruber, 2009: Observing biogeochemical cycles at global scales with profiling floats and gliders prospects for a global array. Oceanography, 22, 216-225, https://doi.org/10.5670/oceanog.2009.81.

Körtzinger, A., J. Schimanski, and U. Send, 2005: High quality oxygen measurements from profiling floats: A promising new technique. J. Atmos. Oceanic Technol., 22, 302-308, https:// doi.org/10.1175/JTECH1701.1.

Legler, D. M., and Coauthors, 2015: The current status of the realtime in situ Global Ocean Observing System for operational oceanography. J. Oper. Oceanogr., 8, s189-s200, https:// doi.org/10.1080/1755876X.2015.1049883.

Liang, Y., and W. Peng, 2010: Minimizing energy consumptions in wireless sensor networks via two-modal transmission. Comput. Commun. Rev., 40, 12-18, https://doi.org/10.1145/ 1672308.1672311.

Marcelloni, F., and M. Vecchio, 2008: A simple algorithm for data compression in wireless sensor networks. IEEE Commun. Lett., 12, 411-413, https://doi.org/10.1109/LCOMM.2008.080300.

—, and — 2009: An efficient lossless compression algorithm for tiny nodes of monitoring wireless sensor networks. Comput. J., 52, 969-987, https://doi.org/10.1093/comjnl/bxp035.

Perry, M. J., B. S. Sackmann, C. C. Eriksen, and C. M. Lee, 2008: Seaglider observations of blooms and subsurface chlorophyll maxima off the Washington coast. Limnol. Oceanogr., 53, 2169-2179, https://doi.org/10.4319/lo.2008.53.5_part_2.2169.

Reineman, B. D., L. Lenain, N. M. Statom, and W. K. Melville, 2013: Development and testing of instrumentation for UAV-based flux measurements within terrestrial and marine atmospheric boundary layers. J. Atmos. Oceanic Technol., 30, 1295-1319, https://doi.org/10.1175/JTECH-D-12-00176.1.

Riser, S. C., and K. S. Johnson, 2008: Net production of oxygen in the subtropical ocean. Nature, 451, 323-325, https://doi.org/ 10.1038/nature06441.

Roemmich, D., S. Riser, R. Davis, and Y. Desaubies, 2004: Autonomous profiling floats: Workhorse for broad-scale ocean observations. Mar. Technol. Soc. J., 38, 21-29, https://doi.org/ 10.4031/002533204787522802.

Sayood, K., 2012: Introduction to Data Compression. 4th ed. Morgan Kaufmann, 768 pp.

Storer, J. A., and T. G. Szmanski, 1982: Data compression via textual substitution. J. Assoc. Comput. Mach., 29, 928-951, https://doi.org/10.1145/322344.322346.

Todd, R. E., W. B. Owens, and D. L. Rudnick, 2016: Potential vorticity structure in the North Atlantic western boundary current from underwater glider observations. J. Phys. Oceanogr., 46, 327-348, https://doi.org/10.1175/JPO-D-15-0112.1.

Wang, N., J. W. Bao, J. L. Lee, F. Moeng, and C. Matsumoto, 2015: Wavelet compression technique for high-resolution global model data on an icosahedral grid. J. Atmos. Oceanic Technol., 32, 1650-1667, https://doi.org/10.1175/JTECH-D-14-00217.1.

Witten, I. H., R. M. Neal, and J. G. Cleary, 1987: Arithmetic coding for data compression. Commun. Assoc. Comput. Mach., 30, 520-540, https://doi.org/10.1145/214762.214771.

Yu, J., A. Zhang, W. Jin, Q. Chen, Y. Tian, and C. Liu, 2011: Development and experiments of the Sea-Wing underwater glider. China Ocean Eng., 25, 721-736, https://doi.org/10.1007/ s13344-011-0058-x.

Ziv, J., and A. Lempel, 1977: A universal algorithm for sequential data compression. IEEE Trans. Inf. Theory, 23, 337-343, https://doi.org/10.1109/TIT.1977.1055714. 